\title{
Methanol Poisoning in A Tertiary Hospital with Analysis of Arterial Blood Gases
}

\author{
Archana Bhat*, Kavina Fernandes, Venkatesh B.m and Prashanth Y.m. \\ Department of Medicine, FR. Muller Medical College, Kankanady-India
}

\begin{abstract}
To study the clinical presentation of all patients admitted to our hospital with the diagnosis of suspected methanol poisoning and to study the correlation between arterial blood gas analysis and outcome in these patients.A total of 6 cases were studied retrospectively during a period of 24 months from Jan 2016 to Dec 2017.All cases were diagnosed in our forensic lab using colour test qualitative method by Schiff ' $\mathrm{s}$ reagent test.The demographic data, clinical manifestations andinitial blood acid - base status and therapeutic interventions done and outcome was studied from the inpatient case records. The data collected was reported as mean, SD, frequency and relative frequency for quantitativedata .Chi-square and fisher' exact test was used between survivors and non- survivors to know their significance. The study was approved by the institutional research and ethics committee. The mean age of the cases in our study was 39.33 years .All were male patients.Breathlessness was seen in all cases (100\%). The mean $\mathrm{pH}$ in our study was 7.27 .The mean HCO3 in our study was 20.25 .The mean PaCO2 was 42.50 . The mean serum creatinine was 2.50 . The mean serum osmolality was 313.83 with high anion gap acidosis(44.70). There was mean base deficit -4.93 . There was mortality $(66.7 \%)$ in our study $.66 .7 \%$ cases required hemodialysis.Analysis of arterial blood gas analysis plays an important role in the early diagnosis of suspected methanol poisoning. Unexplained high anion gap metabolic acidosis with high base deficit play an important role in prognosis .
\end{abstract}

Keywords: Methanol, Arterial Blood Gas Analysis

\section{Introduction}

Methanol poisoning is an important public health problem .It is also known as wood alcohol and it is a cheap and potent adulterant used in manufacture of illicit liquors. Formate is a toxic metabolite of methanol that causes acidosis and inhibits cell cytochrome. The clinical picture of acute methanol poisoning includes headache, dizziness, nausea, vomiting, abdominal pain, visual disturbances, coma, respiratory arrest, seizure, blindness, gastrointestinal haemorrhage and pancreatitis. There are been several episodes of mass methanol poisoning in our country with mass mortality and morbidity rates. ${ }^{1}$.The clinical diagnosis is made in the presence of high index of suspicion of toxic alcohol ingestion, unexplained high anion gap metabolic acidosis and significant osmolar gap ${ }^{2}$.The $\mathrm{pH}$ and high base deficit (BD) reflecting acidosis were important prognostic parameters

Methylated spirit is very cheap and frequently available; hence it is easily adulterated and used as country liquor among poor people who cannot afford ethyl alcohol for their drink. When taken with ethyl alcohol it is metabolized only after complete metabolisation of ethyl alcohol. In course of oxidation, formaldehyde and finally formic acid are formed which are highly toxic. It is associated with serious complications like metabolic acidosis, visual disturbances and neurological deficit. Lactate is produced as formic acid interferes with intracellular respiration and promotes anaerobic metabolism. Both formate and lactic acid contribute to the anion gap increase seen in methanol poisoning. Metabolic acidosis is sometimes refractory and one of the important cause of mortality. ${ }^{4}$ The clinical presentation may vary from anorexia nausea, vomiting, CNS, eye, respiratory and renal toxicity, dyspnoea and respiratory failure may be the clinical presentation resulting from tissue hypoxia and inhibition of cellular respiration by formic acid .Ethyl alcohol is preferentially metabolized by alcohol dehydrogenase resulting in reduced methanol toxicity. Ethanol competitively inhibits the metabolism of methanol to toxic metabolite, formate, by occupying the receptor sites of alcohol dehydrogenase. Fomepizole has been shown to be a potent inhibitor of alcohol dehydrogenase in man.

\section{Materials and Methods}

This is a retrospective study case series study of patients admitted in Father Muller medical college hospital from January 2016 to December2017 who were identified by a positive gastric aspirate test for positive methanol by dipstick method. The diagnosis in all cases was done in our hospital forensic lab using colour test qualitative method by Schiff' s reagent test ${ }^{6}$.Thier levels were not measured as we did not have gas chromatography .The demographic data, clinical manifestations and initial blood 
acid - base status and therapeutic interventions done and outcome was studied from the inpatient case records. A blood acid - base status drawn at admission and outcome was studied. The $\mathrm{pH}$ and $\mathrm{PaCO}_{2}$ and $\mathrm{HCO}_{3}$ level and base deficit (BD) was studied from the initial blood acid base status done at the time of admission with respect to outcome. ${ }^{5}$ The neurological status was noted at the time of admission. The serum potassium and the serum creatinine levels at the time of admission was recorded from the case records at the time of admission Ethyl alcohol was not used intravenously for treatment in this study. Fomepizole is not available locally in our demographic settings and so only hemodialysis was the mode of treatment in our study. The data collected will reported as mean -/+ SD, frequency and relative frequency for quantitative data. Chi- square and fisher' exact test will be used and data will be analyzed with SPSS software version 16 .

\section{Inclusion Criteria}

All patients admitted to the hospital with confirmed methanol poisoning detected from our forensic lab report by SCHIFF's method during Jan. 2016 to Dec.2017.

\section{Exclusion Criteria}

Those patients with toxicology screening test report positive for any other drugs excluding methanol and ethanol.

Study Design: It is a retrospective record based study.

Sample Size: It is a time bound study.

\section{Results}

The mean age in our study was 39.33 years. Their age ranged from 26-60 years. All the patients were male patients. All patients had history of chronic misuse of alcohol. Breathlessness was the most common (83.3\%) clinical presentation in our study as shown in GRAPH 1. Nausea, vomiting and epigastric discomfort was seen in $50 \%$ cases .Respiratory distress was present in all the $(100 \%)$ cases. All the case characteristic are depicted in table 1- table 4.
Analyses of the venous blood gases on admission showed that the median of the $\mathrm{pH}$ was 7.27 with the range of 6.86 - 7.47 . The mean $\mathrm{pH}$ of the patients who died was 7.17 while the mean $\mathrm{pH}$ of the survivors was 7.46 .The median $\mathrm{PaCO} 2$ value in our study was 32.50 The nonsurvivors had a mean $\mathrm{PaCO} 2$ of 48.50 in comparison to the mean $\mathrm{PaCO} 2$ of the survivors was 30.50 .Higher $\mathrm{PaCO} 2$ values were seen in patients who died, although the $\mathrm{p}$ value was .355 not significant. The median HCO3 levels was 18.30 in the study . The patients who died had a lower HC03 levels (19.63) than the patients who survived (21.50) and it was not statistically significant. The median base deficit (BE) in our study was -4 .95.The non survivors had a mean base deficit (BE) -6.70 and the survivors had a mean base deficit (BE) -1.40 and it was not statistically significant. The median anion gap in our study was 45.43 The non survivors had a mean anion gap of 43.89 and the survivors had anion gap of 46.32 .The mean $\mathrm{S}$.osmolality in our study was 313.83 with a minimum value of 281 and a maximum value of 330.The patients who died had a lower serum osmolality (308) in comparison to the survivors (325.50)

Analyses of other lab parameters revealed renal involvement in all our patients included in the study. The mean serum creatinine was 2.50 in the study with no statistically significant difference between survivors (2.17) and non survivors (2.67). The mean serum potassium level was 5.78 Hyperkalemia was seen in all pateints and no statistically significant difference between survivors and non survivors .

Dialysis was required in $66.7 \%$ cases and the most common indication in all these pateints was severe metabolic acidosis .Out of the 4 cases died three patients required hemodialysis as shown in GRAPH 2

The mortality in our study was $66.7 \%$. Out of the 6 cases studied only two patients survived as depicted in GRAPH 3.

Table 1. Patient Characteritic and Outcome.

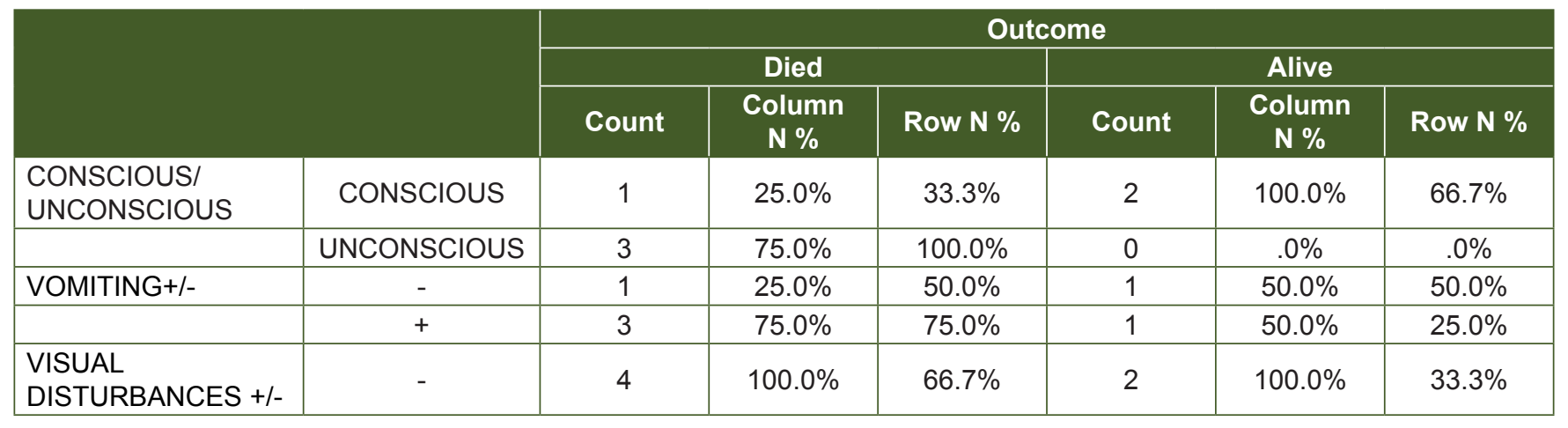




\begin{tabular}{|c|c|c|c|c|c|c|c|}
\hline & & \multicolumn{6}{|c|}{ Outcome } \\
\hline & & \multicolumn{3}{|c|}{ Died } & \multicolumn{3}{|c|}{ Alive } \\
\hline & & Count & $\begin{array}{l}\text { Column } \\
\text { N } \%\end{array}$ & Row N \% & Count & $\begin{array}{l}\text { Column } \\
\text { N } \%\end{array}$ & Row N \% \\
\hline $\begin{array}{l}\text { RESPIRATORY } \\
\text { DISTRESS +/- }\end{array}$ & + & 4 & $100.0 \%$ & $66.7 \%$ & 2 & $100.0 \%$ & $33.3 \%$ \\
\hline \multirow[t]{2}{*}{$\begin{array}{l}\text { ABDOMINAL PAIN } \\
+/-\end{array}$} & - & 2 & $50.0 \%$ & $66.7 \%$ & 1 & $50.0 \%$ & $33.3 \%$ \\
\hline & + & 2 & $50.0 \%$ & $66.7 \%$ & 1 & $50.0 \%$ & $33.3 \%$ \\
\hline \multirow[t]{2}{*}{ DIALYSIS } & - & 1 & $25.0 \%$ & $50.0 \%$ & 1 & $50.0 \%$ & $50.0 \%$ \\
\hline & + & 3 & $75.0 \%$ & $75.0 \%$ & 1 & $50.0 \%$ & $25.0 \%$ \\
\hline $\begin{array}{l}\text { VISUAL } \\
\text { DISTURBANCES +/- }\end{array}$ & - & 4 & $100.0 \%$ & $66.7 \%$ & 2 & $100.0 \%$ & $33.3 \%$ \\
\hline CHEST PAIN & - & 4 & $100.0 \%$ & $66.7 \%$ & 2 & $100.0 \%$ & $33.3 \%$ \\
\hline
\end{tabular}

Table 2

\begin{tabular}{|c|c|}
\hline & Fishers exact test $\mathbf{p}$ \\
\hline CONSCIOUS/UNCONSCIOUS & 0.083 \\
\hline VOMITING+/- & 0.540 \\
\hline VISUAL DISTURBANCES +/- &. \\
\hline RESPIRATORY DISTRESS +/- & 1.000 \\
\hline ABDOMINAL PAIN +/- & 0.540 \\
\hline DIALYSIS &. \\
\hline VISUAL DISTURBANCES +/- &. \\
\hline CHEST PAIN &. \\
\hline
\end{tabular}

Table 3. Analysis of Patient Characteristic with Outcome.

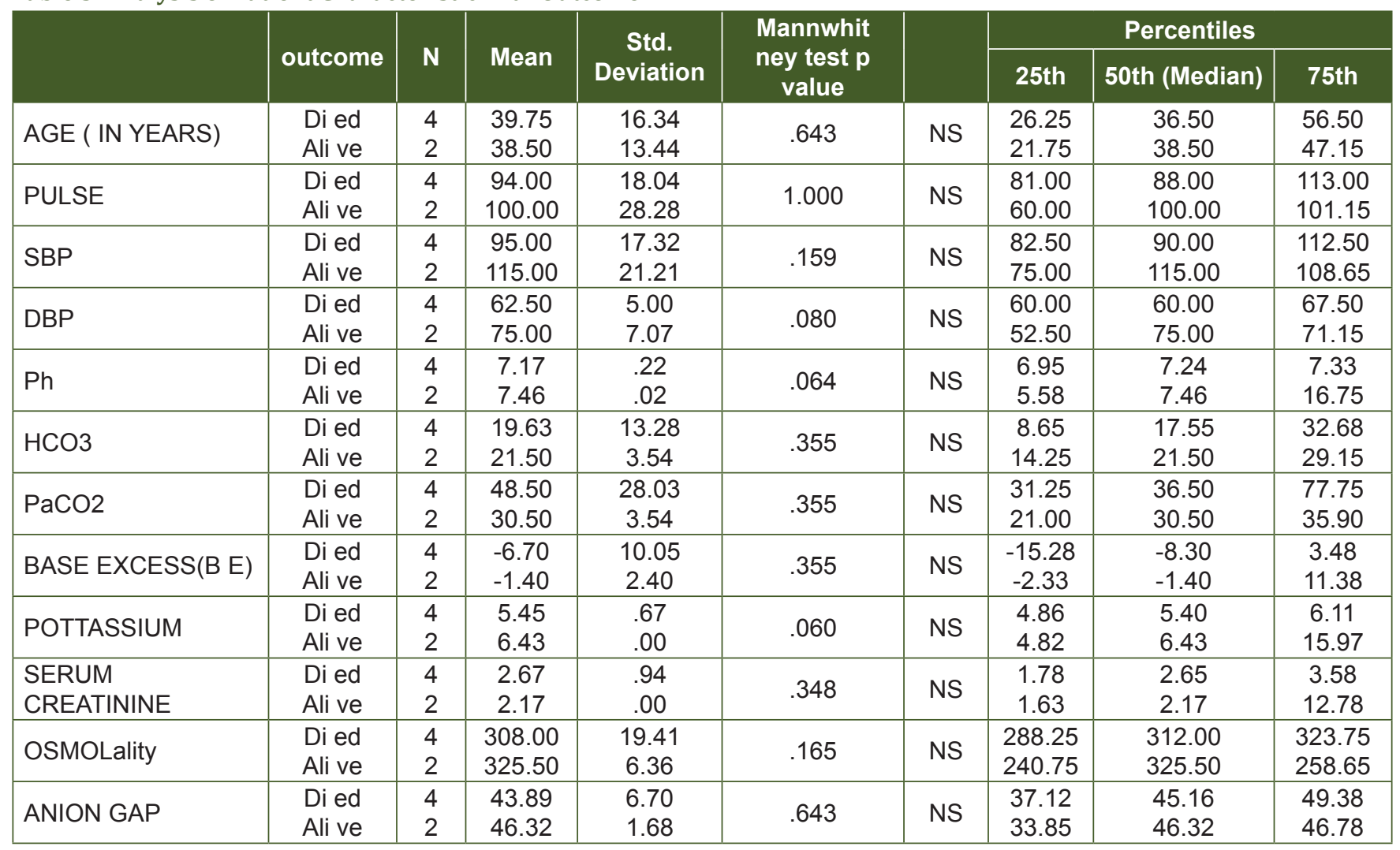


Table 4

\begin{tabular}{|c|c|c|c|c|c|c|c|c|}
\hline & & & & & & & ercentiles & \\
\hline & $\mathbf{N}$ & Mean & Std. Deviation & Minimum & Maximum & 25th & $\begin{array}{c}\text { 50th } \\
\text { (Median) }\end{array}$ & 75th \\
\hline AGE ( IN YEARS) & 6 & 39.33 & 14.02 & 26.00 & 60.00 & 26.75 & 37.50 & 51.00 \\
\hline PULSE & 6 & 96.00 & 19.10 & 80.00 & 120.00 & 80.00 & 88.00 & 120.00 \\
\hline SBP & 6 & 101.67 & 19.41 & 80.00 & 130.00 & 87.50 & 95.00 & 122.50 \\
\hline DBP & 6 & 66.67 & 8.16 & 60.00 & 80.00 & 60.00 & 65.00 & 72.50 \\
\hline $\mathrm{Ph}$ & 6 & 7.27 & .22 & 6.86 & 7.47 & 7.14 & 7.30 & 7.45 \\
\hline $\mathrm{HCO} 3$ & 6 & 20.25 & 10.45 & 5.70 & 37.70 & 14.55 & 18.30 & 27.43 \\
\hline $\mathrm{PaCO} 2$ & 6 & 42.50 & 23.67 & 28.00 & 90.00 & 30.25 & 32.50 & 53.25 \\
\hline BASE EXCESS(B E) & 6 & -4.93 & 8.32 & -17.10 & 6.90 & -11.63 & -4.95 & 1.95 \\
\hline POTTASSIUM & 6 & 5.78 & .72 & 4.82 & 6.43 & 4.93 & 6.02 & 6.43 \\
\hline SERUM CREATININE & 6 & 2.50 & .77 & 1.64 & 3.74 & 2.04 & 2.18 & 3.27 \\
\hline OSMOLality & 6 & 313.83 & 17.77 & 281.00 & 330.00 & 302.75 & 317.50 & 327.75 \\
\hline ANION GAP & 6 & 44.70 & 5.39 & 34.62 & 50.60 & 42.11 & 45.43 & 48.28 \\
\hline
\end{tabular}

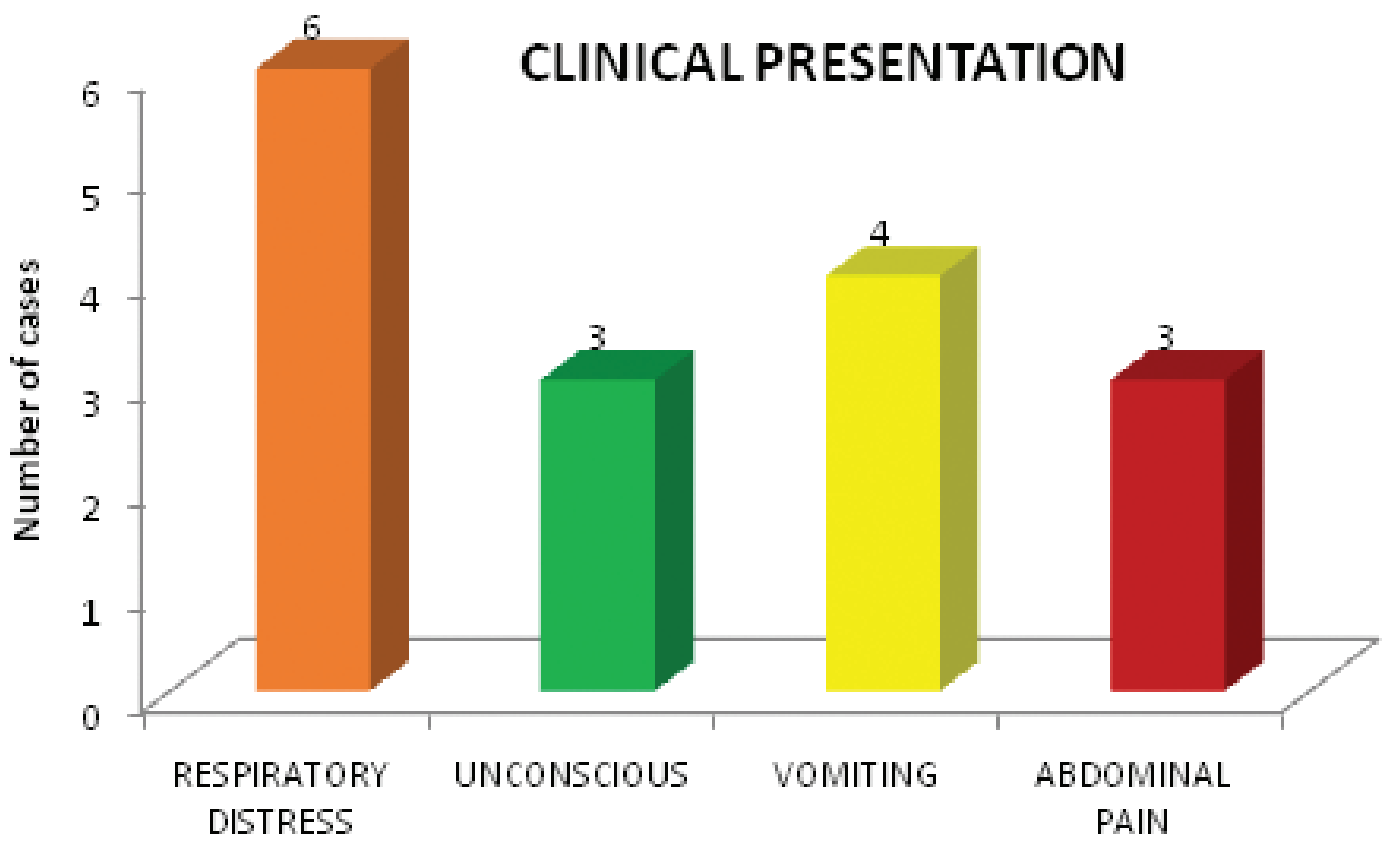




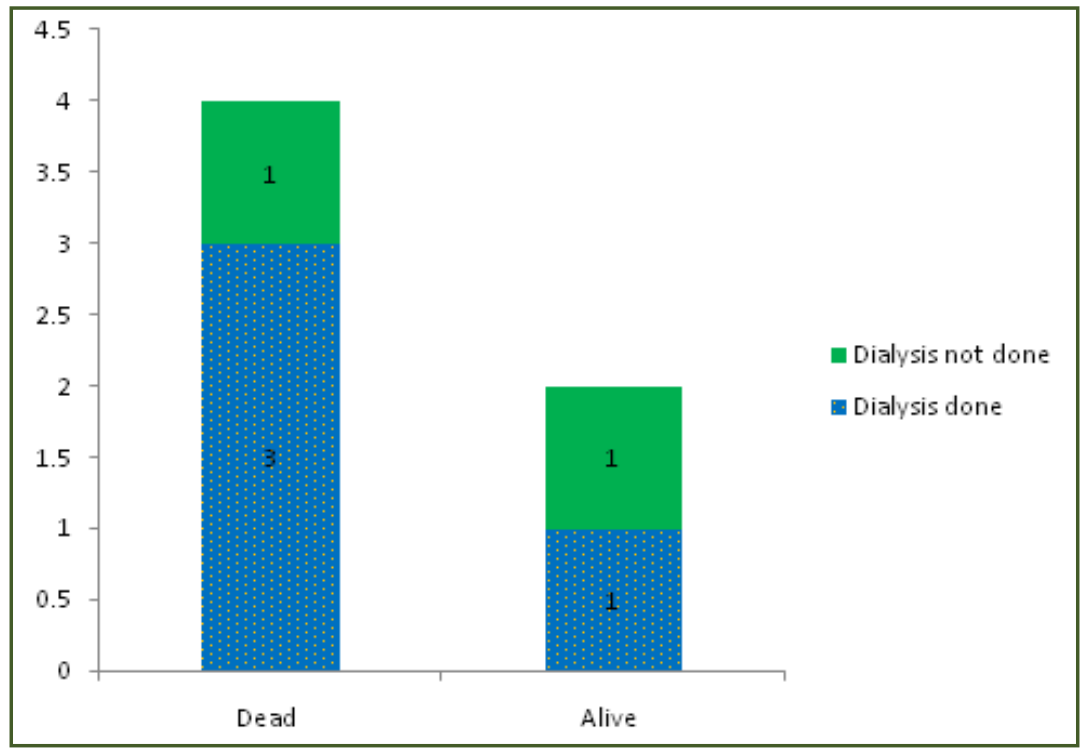

Graph 2 Patients Requiring Dialysis.

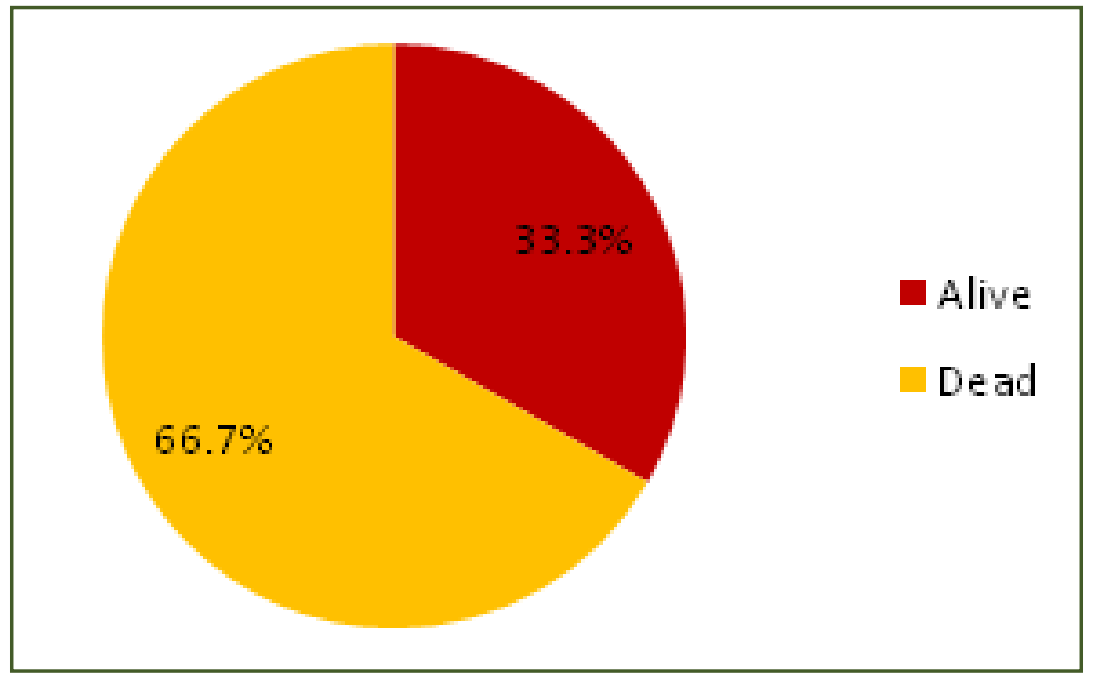

Graph 3 Mortality Analysis.

\section{Discussion}

Although the small number in our case series of methanol poisoning does not carry the statistical weight, it has several important clinical implications.

Out of the six cases studied all were male patients in this study. This can be attributed to the socioeconomic and religion bindings given to the female population in our country.

In a study done by Amin MR ${ }^{3}$ et al all the eight cases studied were male gender. The age ranged from 26-60 years with 3 cases in the third decade indicating more common in the working population . In a study done by Teo SK ${ }^{8}$ in Singapore nausea, vomiting and epigastric discomfort were the common presentation whereas in our study the most common presentation was breathlessness.

The acid - base disturbance seen in this case series study showed low $\mathrm{pH}$ and high anion gap metabolic acidosis with increased osmol gap among the non survivors. There was no correlation between blood $\mathrm{pH}$ and poor prognosis and no statisctically significant $\mathrm{p}$ value between survivors and non survivors . The blood $\mathrm{H}+$ concentration is regulated by $\mathrm{PaCO} 2$ and level of blood $\mathrm{HCO} 3$ and the compensatory mechanism which may not be same in all patient population. The other reason could be the fact that the study is retrospective and the number of patients is relatively small, which is a limitation of this study. 
Higher values of $\mathrm{PaCO} 2$ levels in the arterial blood gas analysis reports have been suggested as a new marker as a prognostic marker in a study done by Shahin ${ }^{9}$. Higher $\mathrm{PaCO} 2$ levels were seen in the non-survivors in our study .Lower $\mathrm{pH}$ values were associated with mortality as per study done by Nizhu N. 1. et a $1^{10}$.The mortality in our study was $66.7 \%$ It was higher as compared to the study done by Moghaddam ${ }^{11}$ where the mortality was $48 \%$ as we did not have fomepizole and ethanol as antidotes due to unavailability in our study.

\section{Conclusion}

Early recognition and rapid intiation of effective therapy is the cornerstone in the management of methanol poisoning. Analysis of arterial blood gases plays an important role in early diagnosis of methanol poisonings . As ethanol and fomepizole are not so available in our settings early intiation of hemodialysis should be instituted. Unexplained high anion gap metabolic acidosis with high base deficit play an important role in prognosis .

\section{Limitations of The Study}

As the sample size was very small, we did not get a significant $p$ value in this study

\section{References}

1. Francis ST, Nair JR, Shiji PV, Mohamed S, Geetha P, et al. (2016) A Case Series of Acute Methanol Poisoning from Northern Kerala. Emergency Med 6:312. doi:10.4172/21657548.1000312

2. Methyl alcohol poisoning: a manifestation of typical toxicity and outcome. Nand L, Chander S, Kashyap
R, Gupta D, Jhobta A. J Assoc Physicians India. 2014 Aug;62(8):756-9.

3. Amin MR, Shohagh AB, Basher A, Rahman M, Faiz MA, Ahasan HA. Methanol Poisoning with Fatality - Case Series in Dhaka Medical College Hospital in Bangladesh . Toxicol Open Access 2017; 3(121):2.

4. Barceloux DG, Bond GR ,Krenzelok EP, Cooper H, Vale JA . American Academy of Clinical Toxicology Practice Guidelines on the treatment of Methanol Poisoning .J Toxicol Clin Toxicol 2002; 40(4): 415-46.

5. Abdolkarim P (2013) Prognostic Factors Including Clinical Manifestation and Paraclinic Finding in Sever Methanol Toxicity. J Alcoholism Drug Depend 1:125. doi:10.4172/2329-6488.1000125

6. Shin JM, Sachs G, Kraut JA. Simple diagnostic tests to detect toxic alcohol intoxications. Transl Res 2008; 152:194-201.

7. Hassanian-Moghaddam H, Pajoumand A, Dadgar SM, ShadniaS: Prognostic factors in methanol poisoning. HumExp Toxicol. 2007, 26: 583-586. 10.1177/0960327106080077.

8. Teo S.K., Lo K.L., Tey B.H. Mass Methanol Poisoning: A Clinico-Biochemical Analysis of 10 Cases. Singap. Med. J. 1996; 37: 485-7

9. Shadnia S, Rahimi M, Soltaninejad K, Nilli A. Role of clinical and paraclinical manifestations of methanol poisoning in outcome prediction. J Res Med Sci 2013;18:865-9.

10. Nizhu N.L.,Barua D., et al .Haemodialysis in Methanol Poisoning .Bangladesh Crit Care J March 2018;6(1): 23-5.

11. Paasma R, Hovda KE, Hassanian-Moghaddam H, Brahmi N, Afshari R, Sandvik L, Jacobsen D. R Risk factors related to poor outcome after methanol poisoning and the relation between outcome and antidotes-a multicenter study. Clin Toxicol 2012; 50:823-831.

*Corresponding author:

Dr. Archana Bhat, Assistant Professor Department Of Medicine, Fr Muller Medical College Mangalore, Kankanady - 575002, Mangalore, India

Email: archanaacharya24@gmail.com

Financial or other Competing Interests: None. 